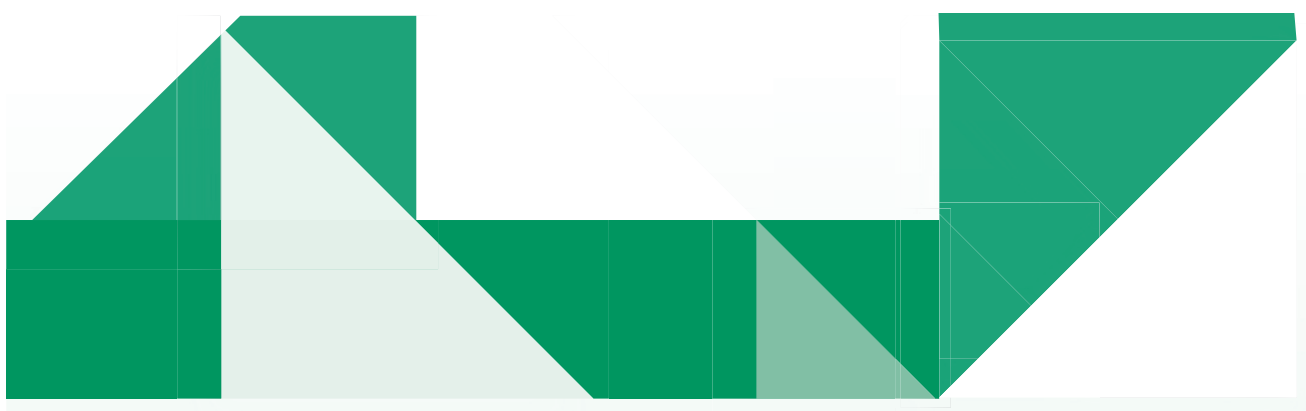

\title{
El diseño de la "reco": una estrategia para iniciar la identificación, la explicitación y el desarrollo del CPC de un tópico de la química de profesores en formación inicial
}

- O projeto "reco": uma estratégia para iniciar a identificação, esclarecimento e desenvolvimento de um tópico cpc química na formação de professores iniciais

- The "core" design: a strategy to start the identification, explicit and development of a topical pck chemistry in initial training teachers

\section{Resumen}

En este estudio se aborda el proceso a través del cual los profesores en formación inicial matriculados en el curso Problemas de la Enseñanza y Aprendizaje de la Química, por orientación reflexiva, comienzan a identificar, explicitar y desarrollar el conocimiento pedagógico del contenido (CPC) hipotético del tópico del currículo de esta disciplina. Para ello, deben enfrentarse a una serie de actividades de aprendizaje circunscritas en los cuatro ámbitos de reflexión del curso, a saber: reflexionar acerca de la enseñanza llevada a cabo por otros a través de los videos de estudios de caso; reflexionar sobre su propia enseñanza a través de sus prácticas educativas; reflexionar sobre las opiniones de los expertos acerca de la enseñanza de las lecturas propuestas en los programas de formación; y reflexionar sobre sí mismos como aprendices de ciencias por medio de actividades científicas. Así, cada una de estas actividades de aprendizaje les suministra a los profesores en formación inicial elementos curriculares e instruccionales para el diseño progresivo de la herramienta metodológica de la representación del contenido (ReCo), la cual recoge el CPC hipotético. La metodología de investigación utilizada en este trabajo es de perspectiva cualitativa e interpretativa por estudio de casos, en la cual la recolección de datos se realiza en tres fases: observación participante de la serie de tareas de aprendizaje que han sido diseñadas, secuenciadas y temporalizadas por el educador de profesores a lo largo del curso; entrevista semiestructurada a los profesores en formación inicial; encuesta y grupo focal. El análisis de dichos datos es orientado por la teoría fundamentada y se centra en las etapas

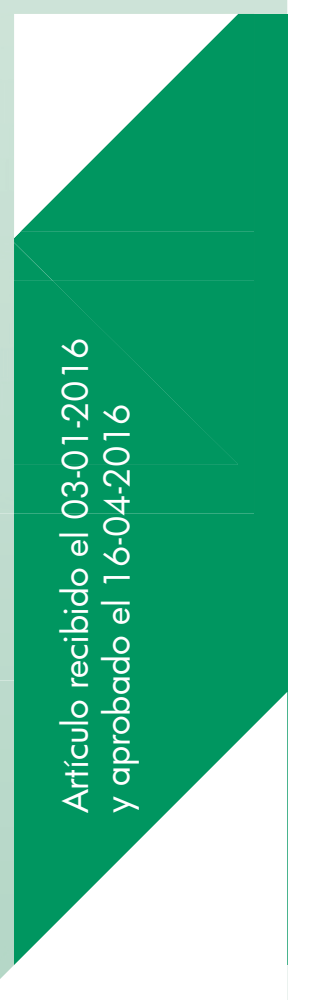

Profesor de la Universidad del Valle (IEP); miembro del grupo de investigación interinstitucional Ciencias, Acciones y Creencias upN-uv (categoría A1).bofeca65@ yahoo.com. ORCID: orcid.org/0000-0002-5833-1975. 
de descripción, ordenamiento conceptual y teorización. Este proceso analítico permite producir generalizaciones naturalísticas, tales como: orientaciones hacia la enseñanza de la ciencia; conocimiento y creencias acerca del currículo de la ciencia; conocimiento y creencias acerca de las estrategias instruccionales para la enseñanza de la ciencia; conocimiento y creencias acerca de la comprensión de los estudiantes de un tópico específico de la ciencia; conocimiento y creencias acerca de la evaluación en la alfabetización científica. Naturalmente, el propósito central de estas generalizaciones descansa en la representación del CPC en cuestión. Finalmente, los resultados de esta investigación han permitido ver que la estrategia de formación que subyace al curso en consideración resulta ser óptima para asistir a los profesores en formación inicial en el inicio de la identificación, la explicitación y el desarrollo del conocimiento pedagógico del contenido hipotético.

Palabras claves

Enseñanza de la química, CPC, profesores en formación, ReCo

\title{
Resumo
}

Este estudo trata de processo através do qual os professores em formação inicial matriculados no curso de "problemas de ensino e aprendizagem de Química" por orientação pensativo, eles começam a identificar, esclarecer e desenvolver o CPC (conhecimento pedagógico do conteúdo) tópico currículo hipotética desta disciplina. Para fazer isso, eles devem enfrentar uma série de actividades de aprendizagem confinados nas quatro áreas de reflexão do curso, a saber: refletindo sobre o ensino realizado por outros, através de estudos de caso de vídeo; refletindo sobre seu próprio ensino através de suas práticas de ensino; refletindo sobre as opiniões dos especialistas sobre o ensino da leitura programas de formação propostos; e refletindo sobre si mesmo como ciências aprendiz por meio de atividades científicas. Assim, cada uma destas actividades de aprendizagem fornece-lhes os professores no currículo de formação inicial e de design instrucional para a ferramenta metodológica progressiva ReCo (Representação de conteúdo), que reflete os elementos hipotéticos CPC. No entanto, a metodologia de pesquisa abordados neste trabalho é o estudo qualitativo e interpretativo caso perspectiva onde a coleta de dados é feita em três fases: Série observação participante das tarefas de aprendizagem que foram concebidos, sequenciado e temporalizada pelo educador de professores ao longo do curso; professores entrevista semi-estruturada em formação inicial; levantamento e grupo focal. A análise destes dados é guiado pela teoria fundamentada concentrando-se nas fases de inscrição, o planejamento conceitual e teorização. Este processo de análise permite produzir naturalista, como generalizações: Orientações para o ensino da ciência; Conhecimentos e crenças sobre o currículo de ciências; Conhecimentos e crenças sobre estratégias instrucionais para o ensino de ciência; Conhecimentos e crenças sobre a compreensão dos alunos sobre um tópico específico da ciência; Conhecimentos e crenças sobre a avaliação da alfabetização científica; é claro, que o objectivo central destas generalizações encontra-se na representação da PCC em questão. Finalmente, os resultados desta pesquisa permitiram ver que a estratégia subjacente ao curso de formação em questão é ideal para ajudar os professores em formação inicial no início da identificação, especificação e desenvolvimento de Pedagógico conhecimento do conteúdo hipotética.

Palavras chaves

Ensino de Química; CPC; formação de professores; ReCo

\begin{abstract}
This study deals with the process through which pre-service teachers enrolled in the course Problems of Chemistry Teaching and Learning, by thoughtful guidance, begin to identify, explicit and develop the hypothetical pedagogical content knowledge (PCK) of the curriculum topic of this discipline. They must face a series of learning activities confined in the four areas of reflection proposed in the course, namely: to reflect on their own learning by means of their educational practice; to reflect on experts 'opinions about the teaching of texts proposed in the programs; and to reflect upon themselves as science learners through science activities.
\end{abstract}


Thus, each of these learning activities provides them with curriculum and instructional elements for the progressive design of Content Representation (CoRe). This methodological tool collects the hypothetical PCK. The research methodology used in this study was qualitative and interpretive perspective case study, in which researchers collected data in three phases: participant observation of the learning tasks series designed sequenced and programmed by teacher trainers throughout the course; semi-structured interview to preservice teachers; survey and focus group. The data analysis follows the grounded theory and focuses on the description, conceptual planning and theorizing stages. This analytical process facilitates the production of naturalistic generalizations, such as: orientations for the teaching of science; knowledge and beliefs about science curriculum; knowledge and beliefs about instructional strategies for science teaching; knowledge and beliefs about students' understanding of a specific topic of science; knowledge and beliefs about scientific literacy assessment. Of course, the main purpose of these generalizations is the representation of the corresponding PCK. Finally, the results of this research show that the strategy underlying the training course of the study is optimal to assist preservice teachers in beginning to identify, specify and develop hypothetical PCK.

Keywords

Chemistry teaching; PCK; pre-service teachers; CoRe 


\section{Introducción}

Los investigadores del campo de la educación en ciencias ha llegado al consenso de que los profesores experimentados y ejemplares poseen un conocimiento profesional que resulta de la mezcla especial entre el contenido disciplinar y la pedagogía general, el cual les permite diseñar e implementar ambientes de aprendizaje centrados en el estudiante (Abell y Bryan, 1997; Bertram y Loughran, 2012). Esta forma de conocimiento fue denominada por Shulman $(1986,1987)$ como conocimiento pedagógico del contenido (CPC), que distingue a los profesores de los especialistas en la disciplina y la pedagogía general, y que se puede desarrollar con la práctica de planear y enseñar una lección determinada.

El modelo del CPC se fundamenta en una perspectiva de enseñanza de naturaleza compleja e incierta que se encuentra mediada por la interacción de diversas bases de conocimiento. En este sentido, se ve esta como una actividad problemática que está configurada por una variedad de decisiones curriculares e instruccionales, cuyo fin es el de asistir a los estudiantes durante el proceso de enculturación.

Ahora bien, desde la génesis del CPC formulado por Shulman, mucho se ha escrito sobre él ya sea para elogiarlo o criticarlo. Sin embargo, el marco teórico que fundamenta el trabajo de Magnusson, Krajcik y Borko (1999), dentro del campo de la educación en ciencias ha ayudado a aclarar y a extender este constructo con el fin de asistir y direccionar los diferentes estudios cuyo foco sea el desarrollo profesional del profesor de ciencias.

Tomando en consideración los anteriores presupuestos, los educadores de profesores afirman que los profesores en formación inicial de química, para planear, enseñar y evaluar efectivamente un tópico de dicha disciplina a lo largo de su carrera profesional deben tener un profundo conocimiento de los diferentes elementos que configuran el CPC de las ciencias. De ahí que los conocimientos que sustentan el CPC deben comenzarse a identificar, explicitar y desarrollar desde los cursos de didáctica de la química. Naturalmente, la experiencia de diseñar e implementar ambientes de aprendizaje resulta clave para la construcción de este conocimiento profesional (Hume, 2010; Hume, 2011 ; Mora y Parga, 2008).

En este sentido, un alto número de profesores en formación entran a los cursos de los programas de educación con un desconocimiento de la diversidad de tensiones a las que se enfrentarán en el momento de aprender a diseñar e implementar ambientes de aprendizaje de un tópico específico. De hecho, no son conscientes de la alta demanda cognitiva que requiere el proceso de planeación y enseñanza de una lección (Loughran, Mulhall y Berry, 2008), además, suelen no apreciar que dicha actividad profesional requiere que el profesor lleve a cabo una serie de razonamientos pedagógicos de orden superior y acciones inteligentes, que solo se desarrollan a través de la experiencia reflexiva (Shulman, 1987). 
Igualmente, la literatura ha reportado que los profesores en formación de ciencias presentan una falta de comprensión conceptual e integrada de las ciencias; de hecho, muchos de ellos entran a los cursos de didáctica de la química con un conjunto de ideas confusas y desconectadas sobre los tópicos específicos (Loughran et al., 2008; Mora y Parga, 2008). Como consecuencia de esta comprensión superficial de la química, tienden a adoptar un modelo de enseñanza centrada en el profesor y los contenidos, donde se entrega de manera unidireccional a los estudiantes una serie de hechos y procedimientos algorítmicos que constituyen un obstáculo para que los estudiantes puedan lograr una comprensión conceptual de las grandes ideas y esquemas conceptuales de la disciplina (Cess-Newsome, 1999).

Los educadores de profesores con el propósito deliberado de comenzar a asistir a los profesores en formación en la identificación, la explicitación y el desarrollo del CPC de la química han aumentado su interés por diseñar cursos de didáctica en los cuales las herramientas metodológicas de la ReCo y los PaP-eR' desempeñan un papel clave en el logro de esa meta (Loughran, Berry, y Mullhall, 2006; Bertram y Loughran, 2012; Candela y Viafara, 2014b). Naturalmente, dichas herramientas fueron diseñadas inicialmente para capturar, documentar y representar el CPC de profesores ejemplares, dado que la interacción entre los contenidos de éstas permiten articular el conocimiento disciplinar, la enseñanza y el aprendizaje de un tópico específico.

Así pues, inicialmente la ReCo fue conceptualizada como la herramienta que permite documentar y representar el CPC de un profesor

ReCo es el acrónimo en español de content representation (representación del contenido); y PaP-eR es el acrónimo de pedagogical and profesional experience repertoire (repertorio de experiencias profesionales y pedagógicas). de ciencias acerca de un tópico específico a través de la solución a los interrogantes que configuran la estructura lógica de esta (véase el anexo 1). Desde luego, el cuerpo de conocimiento de la ReCo encarna las decisiones curriculares e instruccionales que toma el profesor cuando diseña la enseñanza del tópico en consideración. Además, esta permite establecer unas relaciones conscientes entre elementos relacionados con los estudiantes, el contenido específico y la práctica de los profesores (Loughran et al., 2004; Mulhall Berry y Loughran, 2003).

Actualmente, Bertram y Loughran (2012) afirman que el diseño de la ReCo de un tópico específico posee el potencial de andamiar el desarrollo del conocimiento del profesor en tres ámbitos, a saber: práctica profesional, aprendizaje del estudiante y contenido de la disciplina. En el primer ámbito se ha observado que esta apoya al profesor para que reflexione productivamente sobre su práctica educativa, hecho que le permite explicitar su conocimiento intuitivo y tácito acerca de la enseñanza y aprendizaje de las ciencias. El segundo, brinda al profesor la oportunidad para que reflexione sobre el conocimiento del aprendizaje y el aprendiz. Finalmente, el tercer ámbito le permite al profesor profundizar en las diferentes formas de representar y formular un contenido específico a unos estudiantes singulares.

En este sentido, Hume y Berry (2013) se interesaron en el diseño de ambientes de aprendizaje que les brindarán la posibilidad a los profesores en formación de ciencias de comenzar a identificar, explicitar y desarrollar un CPC hipotético sobre un tópico del currículo de química. Para ello, en los cursos de didáctica de la química diseñaron actividades de aprendizaje que les permitieron a los profesores en formación en un comienzo conceptualizar los elementos del CPC, los cuales brindan un marco 
teórico y metodológico para poder reflexionar de manera productiva sobre el CPC que subyace a la ReCo de un profesor ejemplar. Naturalmente, la intención de dichas tareas fue explorar y ganar intuiciones sobre el CPC de profesores de ciencias expertos, con el propósito de que los estudiantes de magisterio se comprometieran en el diseño de una ReCo colectiva de un tema de química. Se destaca que la tarea del diseño de la ReCo fue percibida por los profesores en formación como compleja y tensionante, a causa de la falta de experiencia de enseñanza de tópicos específicos; sin embargo, afirmaron que esta les brinda la oportunidad de iniciar el desarrollo del CPC de la química (Hume y Berry (2013). Por tanto, el estudio deja ver que el andamiaje en la construcción de la ReCo tiene el potencial de comenzar a identificar, explicitar y desarrollar el CPC de la química en profesores novatos (Hume, 2010; Hume, 2011 y Hume y Berry, 2013).

\section{Contextualización}

En la Universidad del Valle (IEP) se rediseñó el curso Problemas de la Enseñanza y el Aprendizaje de la Química, a causa de los desarrollos teóricos y metodológicos actuales que sustentan la línea de investigación del CPC de las ciencias, con el fin de comenzar asistir a los profesores en formación en la identificación, explicitación y el desarrollo del CPC de la química. Para ello, se adoptó un enfoque de enseñanza por "orientación reflexiva" (Abell y Bryan, 1997; Candela y Viafara, 2014a), dado que este se focaliza en la creencia de que aprender a enseñar ciencias es semejante a aprender ciencias por sí mismo. De hecho, es un proceso en el cual el estudiante-profesor a través de una reflexión productiva reevalúa y reformula sus teorías personales sobre la disciplina, la enseñanza y el aprendizaje a la luz de una evidencia perturbadora. Desde luego, la orientación reflexiva genera oportunidades para que los profesores en formación y en ejercicio describan sus ideas, creencias y valores acerca de la enseñanza y el aprendizaje de las ciencias, con el fin de comenzar a aclarar y enfrentar sus teorías personales (Abell y Bryan, 1997).

En este sentido, Abell y Bryan (1997) consideran que un programa de educación de profesores de ciencias fundamentado desde una perspectiva de orientación reflexiva posee una estructura lógica en la cual el estudiante-profesor tiene la posibilidad de aprender a enseñar ciencias a partir del desarrollo de un currículo constituido por cuatro dimensiones, a saber: reflexionar acerca de la enseñanza llevada a cabo por otros a través de los videos de estudios de casos; reflexionar sobre su propia enseñanza a través de sus prácticas educativas; reflexionar sobre las opiniones de los expertos acerca de la enseñanza de las lecturas propuestas en los programas de formación; y reflexionar sobre sí mismo como aprendiz de ciencias por medio de actividades científicas.

Hay que destacar que los cuatro ámbitos de reflexión del curso Problemas de la Enseñanza y el Aprendizaje de la Química están entretejidos y configuran un sistema que suministra una oportunidad singular para que los profesores en 
formación deliberen productivamente sobre las fases del ciclo instruccional (planeación, enseñanza y reflexión) de la enseñanza/aprendizaje de la química. Es decir, estos contextos forman parte de un todo donde cada uno de ellos genera oportunidades para ayudar a los profesores inscritos en los programas de educación a alcanzar un estado de reflexión sobre los elementos que configuran el CPC de la química.

Las anteriores asunciones permiten considerar que las herramientas epistémicas de la $\mathrm{ReCo}$ y los $\mathrm{PaP}-\mathrm{eR}^{2}$ de profesores ejemplares (Candela, 2012; Candela y Viafara, 2014b), pueden cumplir un papel clave dentro del curso Problemas de la Enseñanza y el Aprendizaje de la Química (Universidad del Valle, Cali), cuyo propósito es generar oportunidades para que el estudiante-profesor comience a identificar, explicitar y desarrollar el CPC de la química, dado que estas tienen el potencial de crear un mundo virtual en el que los estudiantes de magisterio pueden experimentar y reflexionar sobre los problemas de la enseñanza y el aprendizaje de un tópico específico del currículo en cuestión. Dicha situación se traduce en la articulación consciente de elementos de la enseñanza, como el conocimiento del contenido, el conocimiento de las dificultades/limitaciones y concepciones alternativas de los estudiantes; el conocimiento de las representaciones y formulaciones de un tópico específico; el conocimiento de las estrategias instruccionales y de evaluación (Candela y Viafara, 2014a).

Finalmente, el educador de profesores que orienta el curso Problemas de la Enseñanza y el Aprendizaje de la Química focaliza la serie

2 La ReCo y los PaP-eRs se consideran herramientas epistémicas, dado que estas permiten documentar y representar un conjunto de conocimientos tanto hipotéticos como en acción acerca de la enseñanza y el aprendizaje de un tópico específico. de actividades de aprendizaje $\mathrm{e}^{3}$ en el diseño de una ReCo de un tópico del currículo de la química, con el propósito de mediar la construcción del CPC hipotético. De hecho, esta estrategia de formación les permite a los profesores en formación comenzar a ganar intuición sobre las bases de conocimientos para la enseñanza que poseen los profesores expertos y ejemplares, las cuales sustentan la toma de decisiones curriculares e instruccionales durante el diseño y la implementación de una enseñanza centrada en el estudiante.

Tomando como referencia los anteriores presupuestos, esta investigación pretende dar respuesta al siguiente interrogante: ¿̇De qué manera la construcción progresiva de una ReCo dentro del marco del curso Problemas de la Enseñanza y el Aprendizaje de la Química contribuye a que los profesores en formación comiencen a identificar, explicitary desarrollar el CPC hipotético de un tópico especifico del currículo de la química?

\section{Aspectos metodológicos de la investigación}

Esta investigación se lleva a cabo por medio de una metodología cualitativa de estudios de caso, la cual tiene como fin comprender en profundidad el caso en cuestión (Stake, 1999). Naturalmente, las interacciones dialógicas entre los diferentes agentes del caso desempeñan un papel clave para darle solución al problema, es decir, el educador de profesores, los profesores en formación y el investigador relatan su historia, dentro de una transacción de significados. Así, los datos generados en estas interacciones son registrados en notas

\footnotetext{
3 Se destaca que las actividades de aprendizaje han sido seleccionadas, secuenciadas y programadas a lo largo de los cuatro ámbitos de reflexión que configuran el curso por "orientación reflexiva".
} 
de campo de las experiencias compartidas, transcripciones de las entrevistas de las discusiones y acuerdos de los participantes.

Se destaca que ocho estudiantes se matricularon en el curso, cuatro de ellos pertenecen al programa de Licenciatura en Básica Secundaria con Énfasis en Educación en Ciencias, en tanto que los demás provienen del programa de Química Pura de la Facultad de Ciencias Experimentales ${ }^{4}$. Conviene señalar que, a pesar de los diferentes antecedentes de los dos grupos de estudiantes, el propósito central del curso es el mismo para todos (identificar, explicitar y desarrollar el CPC hipotético de un tópico de la química). Sin embargo, con el fin de llevar a cabo esta investigación se seleccionaron dos estudiantes que actualmente se encuentran cursando la Licenciatura en Educación Básica con Énfasis en Educación en Ciencias Naturales de la Universidad del Valle, los cuales formarían el caso de estudio bajo consideración.

Ahora bien, en la presentación del curso Problemas de la Enseñanza y el Aprendizaje de la Química se les comunica a los profesores en formación que el contenido y la metodología de este, junto con sus razonamientos y acciones pedagógicas serán el centro de estudio y recolección de datos para esta investigación. Para ello, el educador de profesores que orienta el curso afirma que las diferentes tareas que se aborden durante este serán evaluadas de manera formativa, con la intención de que quienes participen como sujetos-investigados del estudio bajo consideración se sientan cómodos y puedan intervenir a lo largo de los ámbitos reflexivos de manera natural y abierta. Como era de esperarse, a partir de dicha aclaración los profesores en formación fueron conscientes del papel clave que desempeñaban en el desarrollo del estudio, de ahí que se comprometieran a llevar a cabo las diferentes tareas de investigación, las cuales requieren de niveles altos de inversión de cognición y tiempo. Además, consideraron que el participar activamente en este estudio era una excelente oportunidad para comenzar a construir hipotéticamente el conocimiento profesional del magisterio.

Por otro lado, la selección del caso de este estudio (las dos profesoras en formación) se llevó a cabo a partir de los siguientes criterios: (a) rentabilidad de lo que podemos aprender de los sujetos estudiados, es decir, se tuvo en cuenta qué casos pueden ayudarnos a comprender y a producir asertos de la identificación, explicitación y desarrollo del CPC hipotético de la química en profesores en formación; (b) tiempo disponible para el trabajo de campo; (c) acceso a los sujetos-actores del caso y (d) sujetos-actores dispuestos a invertir tiempo y espacio en el desarrollo de la investigación.

Teniendo en cuenta los anteriores criterios se procedió a comprometer a dos estudiantes del curso Problemas de la Enseñanza y el Aprendizaje de la Química, quienes permitirán ver el proceso a través del cual se inician la identi-

4 Los ocho profesores en formación actualmente están matriculados en el octavo semestre. 
ficación, la explicitación y el desarrollo del CPC hipotético de un tópico específico por medio del diseño de la herramienta de la ReCo. Así pues, Yuly y Vanessa (estudiantes matriculadas en el programa de licenciatura), tomaron la decisión de participar de manera explícita en esta investigación. Para ello, llevaron a cabo todas las actividades propuestas por el investigador ( $\mathrm{CoRe}^{5}$, entrevista, encuesta, entre otras). Sin embargo, los seis estudiantes restantes también realizaron la gran mayoría de las actividades formuladas por el educador de profesores. Se destaca que los resultados de esta investigación provienen en un alto porcentaje de las fuentes documentales producidas por las dos estudiantes antes mencionadas.

\section{Análisis de datos y codificación}

La gran cantidad de información recogida desde las respectivas fuentes documentales se analiza tomando como referente teórico la teoría fundamentada de Strauss y Corbin (2002), la cual está configurada en tres fases: descripción, ordenamiento conceptual y teorización. En este sentido, se llevó a cabo una codificación abierta, selectiva y axial, cuyo principal fin era generar una teoría que le diera solución al problema en cuestión.

Para realizar dicha tarea analítica, se revisó de manera sistemática la ReCo totalmente diseñada por las dos profesoras en formación con el propósito de recoger evidencia que apoyara la identificación, la explicitación y el desarrollo de los componentes del CPC hipotético de un tópico específico del currículo de la química. Por tanto, se revisó detalladamente el contenido de cada uno de los ítems de las diferentes versiones de las ReCo diseñadas, y

5 Deseamos subrayar que Yuly y Vanessa comenzaron a identificar y desarrollar el CPC de la química, la primera lo hizo para el contenido de "enlaces químicos", en tanto que la segunda lo realizó en el tópico "propiedades de los sólidos, líquidos y gases". se compararon con las unidades de análisis con sentido independiente provenientes de las otras fuentes documentales.

El proceso de codificación realizado a la información contenida en las fuentes documentales de las ReCo, entrevista, encuesta, hojas de reflexión, relatos narrativos y grupo focal se llevó a cabo desde los marcos teóricos del CPC provenientes de la perspectiva de Magnusson et al. (1999). Esta tarea permitió visualizar las categorías analíticas de este estudio, el CPC y la ReCo (véase la tabla 1). Se destaca que estas, junto con las subcategorías, poseen una naturaleza deductiva, es decir provienen de la literatura basada en la investigación.

Desde luego, el CPC para este estudio es considerado la categoría central o medular, que articula de manera semántica las subcategorías que configuran la categoría de la ReCo (véase la tabla 1). Es decir, el CPC tiene la capacidad de vincular proposicionalmente las unidades de análisis adscritas a sus subcategorías con las de la categoría de la ReCo. Como consecuencia de esta situación, se produce una serie de generalizaciones naturalísticas, las cuales se desarrollan con el fin de generar un marco teórico que brinde la posibilidad de describir y comprender la identificación, la explicitación y el desarrollo del CPC de un tópico del currículo de la química en los profesores en formación en cuestión.

Finalmente, el análisis de esta información tuvo como fin central el de buscar elementos empíricos que sustentaran la identificación, la explicitación y el desarrollo de los elementos de un futuro CPC de un tópico específico como resultado del diseño de la ReCo desde un enfoque por "orientación reflexiva". Además, este análisis buscó el nivel de independencia y competencia por parte de los profesores en formación cuando ubican información apropiada para el diseño de su ReCo. 
Tabla 1. Relación selectiva entre las categorías del CPC y la ReCo.

\begin{tabular}{|c|c|c|}
\hline \multirow{6}{*}{$\begin{array}{l}\text { CATEGORÍA } \\
\text { CENTRAL } \\
\text { CPC }\end{array}$} & SUBCATEGORÍAS & $\begin{array}{c}\text { CATEGORÍA } \\
\text { ReCo }\end{array}$ \\
\hline & $\begin{array}{l}\text { Orientaciones hacia la enseñanza de la } \\
\text { ciencia }\end{array}$ & $\begin{array}{l}\text { 2. ¿̇Por qué es importante que los alumnos sepan esta idea? } \\
\text { 7. ¿̇Cuáles procedimientos de enseñanza emplea? (y las razones } \\
\text { particulares de su uso con esta idea) }\end{array}$ \\
\hline & $\begin{array}{l}\text { Conocimiento y creencias acerca del } \\
\text { currículo de la ciencia }\end{array}$ & $\begin{array}{l}\text { 1. ¿Qué intenta que aprendan los alumnos alrededor de esta } \\
\text { idea? } \\
\text { 3. ¿Qué más sabe respecto a esta idea (y qué no incluye en sus } \\
\text { explicaciones a sus alumnos)? }\end{array}$ \\
\hline & $\begin{array}{l}\text { Conocimiento y creencias acerca de } \\
\text { las estrategias instruccionales para la } \\
\text { enseñanza de la ciencia. }\end{array}$ & $\begin{array}{l}\text { 7. ¿̇uáles procedimientos de enseñanza emplea? (y las razones } \\
\text { particulares de su uso con esta idea) }\end{array}$ \\
\hline & $\begin{array}{l}\text { Conocimiento y creencias acerca de la } \\
\text { comprensión de los estudiantes de un tópico } \\
\text { específico de la ciencia }\end{array}$ & $\begin{array}{l}\text { 4. ¿̇ } \text { Cúles son las dificultades/limitaciones relacionadas con la } \\
\text { enseñanza de esta idea? } \\
\text { 5. ¿̇Qué conocimientos acerca del pensamiento de los alumnos } \\
\text { influyen en su enseñanza de esta idea? } \\
\text { 6. ¿̇Qué otros factores influyen en su enseñanza de esta idea? }\end{array}$ \\
\hline & $\begin{array}{l}\text { Conocimiento y creencias acerca de la } \\
\text { evaluación en la alfabetización científica }\end{array}$ & $\begin{array}{l}\text { 8. ¿QQué formas específicas de evaluación del entendimiento o } \\
\text { de la confusión de los alumnos emplea alrededor de esta idea? }\end{array}$ \\
\hline
\end{tabular}

Fuente: elaboración propia

\section{Presentación de los resultados}

El proceso de codificación abierta, axial y selectiva que se le realizó a la extensa información contenida en el conjunto de fuentes documentales permitió establecer una serie de generalizaciones naturalísticas. Estas están alineadas con las subcategorías de la categoría del CPC circunscrita a la perspectiva de Magnusson et al. (1999) (véase la tabla 1), además se vincularon semánticamente con las subcategorías que configuran la ReCo. Estos vínculos fueron representados a través de proposiciones y macroestructuras textuales, que son el mecanismo por medio del cual se materializó la teoría generada desde el proceso de ordenamiento conceptual.

Así pues, la teoría que describe y explica el proceso a través del cual los profesores en formación de ciencias matriculados en el curso Problemas de la Enseñanza y el Aprendizaje de la Química comenzaron a identificar, explicitar y desarrollar los elementos del CPC que encarnan las decisiones curriculares e instruccionales de la enseñanza de la química es la orientación hacia la enseñanza de la ciencia.

\section{Orientación hacia la enseñanza de la ciencia}

Este elemento del CPC de la química se visualiza dentro de la información contenida en las fuentes documentales, las cuales abordan elementos como los propósitos educativos y los diferentes enfoques instruccionales. Es decir, el establecimiento de la relación entre dichos elementos permite evidenciar tanto el modelo de enseñanza con el que entra el estudiante-profesor al curso de didáctica de la química, como el desarrollo progresivo de este aspecto a lo largo del curso en cuestión. 
En este sentido, a pesar de que los profesores en formación han construido un marco teórico que recoge las diferentes teorías del aprendizaje (ej., conductista, procesamiento de la información, constructivismo cognitivo y sociocultural) durante los cursos previos de didáctica de las ciencias, sostienen en un alto porcentaje un enfoque de enseñanza centrado en el profesor y los contenidos, sin ser conscientes de las teorías del aprendizaje que fundan dicho enfoque.

De hecho, los profesores en formación en el diseño de las primeras ReCo no fueron conscientes del tipo de problema de la enseñanza y de la naturaleza del contenido. Por ello, diseñaron actividades de aprendizaje en las que por la naturaleza del problema de enseñanza y del contenido era más pertinente focalizar el proceso de instrucción en el profesor y el contenido. Así pues, esta decisión de diseño debía estar sustentada por teorías del aprendizaje del procesamiento de la información, las cuales permiten organizar y presentar la información para que los estudiantes logren una articulación entre esta y los marcos que ya presentan en su memoria permanente. Desde luego, esto se traduce en una desarticulación entre las metas de aprendizaje y las diferentes estrategias y modelos de enseñanza.

En el inicio del curso no era consciente de la existencia de la relación que debe de existir entre las diferentes teorías del aprendizaje y la naturaleza del tema y la enseñanza de este. Por ello, en un comienzo diseñé actividades de aprendizaje donde el profesor explicaba los principales aspectos del tema y los estudiantes solo se dedicaban a poner atención, sin haber hecho un análisis previo de cuál teoría de aprendizaje se ajusta más a la enseñanza del tema. (Entrevista, Vanessa)
Ahora bien, los profesores en formación apoyados por el educador de profesores y sus compañeros a lo largo de la socialización de las diferentes $\mathrm{ReCo}$, de manera progresiva fueron tomando conciencia de la relación estrecha entre las metas y propósitos de enseñanza, los contenidos, la naturaleza del problema de enseñanza y el enfoque de instrucción. Es decir, comenzaron a visualizar que no todos los contenidos de la química se deben diseñar y enseñar a partir de un enfoque constructivista cognitivo o sociocultural (Christensen, 2008).

Las actividades de desarrollo profesional adscritas a los diferentes ámbitos de reflexión propuestos por el curso Problemas del Aprendizaje y la Enseñanza de la Química permiten a los profesores en formación comenzar a establecer una relación entre los diferentes tópicos del currículo de la química y su naturaleza en conjunción con el problema de la enseñanza. Así pues, ellos lograron visualizar que cuando un tópico es demasiado abstracto quizás no se puede encontrar una situación problema cercana al mundo del estudiante, con la intención de representarlo y formularlo. En este sentido, se debe tomar la decisión de diseñar una actividad de aprendizaje centrada en el profesor y los contenidos (ej., animaciones, simuladores, entre otros). Ahora bien, en el momento en que se logre vincular el tópico en cuestión con aspectos del mundo de la vida, entonces se puede diseñar una actividad centrada en el estudiante. La discusión sobre las situaciones problema sería el medio a través del cual comenzaría a emerger en el aula el tópico en consideración.

Al respecto, la orientación de la enseñanza que se adopte durante el diseño de un ambiente de aprendizaje puede depender de variables como la naturaleza del tópico, el problema de enseñanza, los antecedentes de 
los estudiantes y los recursos, entre otros. Este presupuesto se ve reflejado en el siguiente testimonio:

El proceso de la socialización de las ReCo es una buena estrategia, porque permite que uno a través de la discusión comience a conocer de la relación que existe entre la naturaleza del tema a enseñar y las diferentes teorías del aprendizaje. Es decir, que brindó la posibilidad para ver cuando podemos crear actividades de aprendizaje centradas en el profesor y cuando centradas en el estudiante. (Grupo focal)

\section{Conocimiento y creencias acerca del currículo de la ciencia}

En cuanto a este elemento del CPC de la química, se observa que a lo largo del diseño de las primeras ReCo, los profesores en formación descomponen el contenido central en una serie de ideas fragmentadas y desconectadas. Estas no recogían el conocimiento sustantivo que subyace a los principales elementos teóricos del contenido en consideración, y fueron representadas por medio de un rótulo o título sin entrar a formular de manera proposicional y sintética la idea en cuestión, como se evidencia a continuación:

La mayor dificultad que he tenido para elaborar la ReCo es el desconocimiento del contenido enlace químico. De hecho, poseo un conocimiento limitado e impreciso de esta idea. En efecto, esta dificultad quedó expuesta al momento de seleccionar y secuenciar las grandes ideas que representan el tema específico. Sumado a esto, cuando respondí las preguntas 1, 2, y 3 de la ReCo para cada una de las ideas, las respuestas no fueron inmediatas, me demoré casi dos semanas. Esta dificultad hizo que reflexionara respecto a mi formación y futuro desempeño docente, llegando a la conclusión que mi CPC respecto al tema es muy vago para enseñarlo a estudiantes de secundaria. En este orden, el conocimiento que yo le enseñe a un estudiante será superficial y no le permitirá una formación significativa y "sólida" que le contribuya a comprender conocimientos de mayor nivel de complejidad. (Diario reflexivo, Yuly)

Ahora bien, a medida que las ReCo fueron refinadas por los profesores en formación de manera colegiada, ellos comenzaron a ser conscientes de que esta ideas no recogían el contenido real del tópico que estaban representando, por eso tomaron la decisión de retornar a los libros universitarios y escolares con el propósito de comprender el tema con el fin de volverlo enseñable a unos estudiantes particulares. Esta tarea les brindó la oportunidad de seleccionar, secuenciar y programar las ideas claves que estructuran el contenido con el fin de que unas ideas apalanquen el aprendizaje por comprensión de las otras.

Adicionalmente, la comprensión del contenido de la química por parte de los profesores en formación permitió que lograran vincular cada una de las ideas que configuran dicho contenido de manera consciente con las diferentes metas de aprendizaje y enseñanza propuestas en el currículo prescripto por el 
Estado. Esta articulación desempeña un papel clave a lo largo del diseño de materiales instruccionales ya que direcciona la construcción de la secuencia de actividades, lo cual brinda al aprendiz la oportunidad de construir progresivamente el contenido del currículo de la química. Este presupuesto se refleja en las siguientes afirmaciones:

Umm, un aspecto difícil para nosotros fue la selección y secuenciación de las grandes ideas, ya que, tenemos un conocimiento de la química muy flojito, así que, las dos primeras preguntas de la ReCo para poder darle respuesta medianamente bien, nos tocó acudir a los libros universitarios y a las explicaciones del profesor del curso. Adicionalmente, tuvimos en cuenta los estándares básicos de competencia en ciencias naturales y educación ambiental los cuales encarnan los propósitos de este currículum con el fin de ver en cuales de estos estaban ubicados las grandes ideas seleccionadas. Comenzamos a considerar que los estándares en compañía de los temas nos podría [sic] ayudar a crear las actividades de aprendizaje. (Grupo focal)

Otro aspecto que dejan ver las primeras ReCo hace referencia al currículo vertical y horizontal. El bajo nivel del conocimiento del contenido disciplinar restringe la vinculación del tópico en cuestión con los otros contenidos dentro y a lo largo de los grados, es decir que los profesores en formación entran al curso Problemas de la Enseñanza y el Aprendizaje de la Química sin un conocimiento curricular vertical y horizontal de la materia. La anterior restricción comienza a ser superada en el momento en que los profesores en formación internalizan cada una de las ideas que configuran el tópico en cuestión del currículo de la química gestionándolas. Así pues, comienzan a visualizar las diferentes conexiones intra- e intercurriculares que existen entre los tópicos del currículo con el propósito de representarlos por medio de actividades de aprendizaje. Esta teoría se evidencia en la siguiente afirmación:

Ah, sí, si claro tiene sentido porque las ideas deben ir, como de menor nivel a mayor nivel, a pesar de que, todas las ideas hacen parte de un mismo tópico, las ideas son subsensoras, la primera es subsensora de la segunda, la segunda de la tercera y la tercera de la cuarta. Por tanto, es necesario que se haga una secuenciación, que exista un orden entre las diferentes ideas, porque no es lo mismo empezar por fuerzas intermoleculares y luego seguir con electronegatividad, y luego seguir con la polaridad de la molécula y luego con conceptualización del enlace iónico. Entonces, sí es necesario que exista un orden y una secuenciación en las ideas. (Entrevista, Vanessa)

Conocimiento y creencias acerca de las estrategias instruccionales para la enseñanza de la ciencia

El conocimiento de las estrategias instruccionales es quizás uno de los elementos más importantes que configuran el constructo del CPC, ya que, este recoge los otros aspectos de dicha base de conocimiento. En este sentido, esta especie de conocimiento direcciona el diseño de las diferentes actividades de aprendizaje, las cuales brindan la posibilidad al estudiante para construir de manera colegiada y progresiva cada una de las grandes ideas que configuran el tópico bajo consideración.

Ahora bien, los profesores en formación al entrar al curso Problemas de la Enseñanza y el Aprendizaje de la Química dejan ver un bajo nivel de conocimiento de las estrategias instruccionales y actividades de aprendizaje para la enseñanza de las ciencias. De hecho, no contestan el ítem que recoge esta clase de 
conocimiento en las primeras $\mathrm{ReCo}$, o simplemente se limitan a enumerar una serie de interrogantes sin un contexto problemático. Quizás una de las causas de esta restricción en esta base de conocimiento sea que los profesores en formación no logran articular los componentes disciplinar y pedagógico (general y específico a una disciplina) del programa de educación, situación que obstaculiza el diseño de ambientes de aprendizaje que apoyen el aprendizaje por comprensión conceptual, como lo sugiere la siguiente afirmación:

En las primeras ReCos se me dificultó mucho la construcción de actividades de aprendizaje, ya que no manejo el conocimiento de la disciplina de la química, en segundo lugar, el conocimiento de la pedagogía de dicha disciplina también es muy bajo, de eso he tomado conciencia durante la elaboración de mi ReCo. (Entrevista, Vanessa)

Con la intención de comenzar a superar la anterior restricción, el educador de profesores programó una serie de sesiones en las cuales los profesores en formación reflexionaban desde los cuatro ámbitos que configuran el curso en cuestión por "orientación reflexiva". Para ello, se recurrió al siguiente material curricular: artículos sobre la enseñanza y el aprendizaje de la química; video de casos; actividades experimentales; artículos sobre el diseño de ambientes de aprendizaje; ReCo y PaP-eR de profesores ejemplares, entre otros.

De hecho, esta tarea les permitió a los profesores en formación comenzar a identificar y explicitar bases del conocimiento, tales como: contenido disciplinar, dificultades y concepciones alternativas; estrategias y modelos de enseñanza (ej., $\mathrm{POE}^{6}$, ciclo de aprendizaje), formas de representar y formular un tópico. Se destaca que las actividades de desarrollo profesional que ellos enfrentaron estuvieron secuenciadas y programadas a lo largo del proceso del diseño de las diferentes $\mathrm{ReCo}$, con el fin de que el conocimiento adquirido desde estas sirviera de insumo para el refinamiento y rediseño de las diversas ReCo.

Esta estrategia de desarrollo profesional les brindó la oportunidad a los profesores en formación de comenzar a identificar e internalizar el contenido de las anteriores bases de conocimiento, las cuales les ayudaron a direccionar el diseño y la secuenciación del conjunto de actividades de aprendizaje. Además, estos factores direccionan los razonamientos de los futuros profesores en cuanto a las estrategias instruccionales más apropiadas de acuerdo con la naturaleza de la gran idea a representar y el problema de enseñanza de esta, el cual se materializa en la toma de decisiones de diseño.

Por ejemplo, el tener claro el contenido que subyace a la idea de la electronegatividad, el propósito de aprendizaje vinculado con las posibles dificultades y concepciones alternativas (ReCo Yuly) permitió que la estudiante profesora Yuly decidiera apoyarse en los recursos digitales para representar la idea de la

6 POE es el acrónimo de la estrategia de enseñanza de las ciencias denominada Predecir, Observar y Explicar. 
electronegatividad, en conjunción con la serie de tareas problema que, al ser resueltas de manera colegiada, posibilitan que comience a emerger la gran idea bajo consideración: "Actividad 1 Ingresa al siguiente link https:// www.youtube.com/watch? $v=Q_{c} U y 5 n O E n V 0$, y observa el video hasta el minuto 2:37. Partiendo de la información contenida en éste responde:..." (ReCo, Yuly)

El análisis de las primeras ReCo diseñadas por los profesores en formación dejó ver que la mayoría de las actividades de aprendizaje estaban estructuradas únicamente por una serie de interrogantes o problemas, sin estar circunscritos a un contexto problemático u organizador previo (noticia, situación sociocientífica, gráficas, animaciones, simulaciones, videos, líneas de tiempo, laboratorio, demostraciones, historietas, entre otros). Por ejemplo, las primeras actividades de aprendizaje diseñadas por Vanessa estuvieron limitadas a interrogantes como: "¿̇La presión influye en los puntos ebullición?". Posteriormente, ellos comenzaron a hacer evidente la necesidad de diseñar contextos problemáticos, cuyo fin central es generar en los estudiantes la necesidad del aprendizaje y activar sus conocimientos previos y concepciones alternativas.

Se destaca que el desarrollo gradual de esta base de conocimiento les permitió a los profesores en formación identificar y explicitar el aspecto de la gestión y la administración del aula. De hecho, en un inicio solo utilizaron estructura de aula no interactiva, en la cual el profesor transmite un conocimiento. Sin embargo, el diseño de las diversas ReCo y el estudio de la literatura sobre las técnicas y estrategias de enseñanza de la química les

7 Por razones de espacio en este documento no se anexan las diferentes ReCo de las dos estudiantes, donde se puede evidenciar el desarrollo progresivo del CDC de un tópico. Si desea consultarlas, estas se hallan en el informe final de estudio, el cual se encuentra en la biblioteca de la Universidad del Valle. brindaron la posibilidad de conocer organizaciones de aula, como pequeños grupos de discusión, discusión con toda la clase y trabajo individual. De hecho, dichas estructuras de aula han sido consideradas por la comunidad de educadores de profesores como un medio a través del cual se ayuda a los estudiantes para que alcancen un aprendizaje por comprensión conceptual, segúnse evidencia a continuación:

Para ello, el docente organiza el aula en una estructura de discusión con toda la clase y se analiza la información y los ejemplos sobre los diagramas de fases, haciendo especial énfasis en que los estudiantes logren interpretar cómo dependiendo de las condiciones de presión y temperatura, una sustancia la podemos encontrar en estado gaseoso, líquido o sólido.

Una vez se haya explicado y comprendido el significado y función de los diagramas de fases, se procederá a aplicar dichos conocimientos. Para ello, el docente organiza la clase en pequeños grupos de discusión, en donde a cada grupo se le entregará un tipo de sustancia con sus respectivos puntos de fusión y ebullición en diferentes condiciones ambientales, por lo que ellos deberán determinar cuáles de los diferentes diagramas de fases que se les muestra representa mejor el comportamiento de su sustancia. (Diario reflexivo, Vanessa)

\section{Conocimiento y creencias acerca de} la comprensión de los estudiantes de un tópico específico de la ciencia

Este elemento del CPC de la química fue el de menor nivel de elaboración en las primeras $\mathrm{ReCo}$ diseñadas por los profesores en formación, de hecho, no llenaron este ítem en la estructura de la ReCo. Quizás esto obedezca a la falta de experiencia de enseñanza que 
tienen los futuros profesores, así como a la desarticulación que se presenta entre los contenidos de la disciplina, la psicología y los métodos de enseñanza de tópicos específicos.

Sin embargo, la continua discusión colegiada ante el colectivo de aula de las diferentes ReCo en conjunción con el andamiaje llevado a cabo por el educador de profesores les brindó la posibilidad a los profesores en formación de comenzar a explicitar y desarrollar el conocimiento sobre las dificultades y concepciones alternativas de la química y su enseñanza que tienen los profesores en formación inicial. El otro medio que asistió el desarrollo de este conocimiento fue la lectura reflexiva de la literatura que se focaliza en recoger las dificultades y concepciones alternativas que presentan los estudiantes sobre la química. Esta asunción se puede evidenciar a través de la afirmación siguiente:

... escogí el tópico del enlace químico para diseñar la enseñanza de éste, pero reconozco que además del contenido que constituye el tema de enlace químico, también he tenido dificultad en encontrar las dificultades [sic] y posibles concepciones alternativas con las que llegan los estudiantes. De ahí que, por asesoría de mi profesor, decidí consultar la literatura acerca de las concepciones alternativas sobre el enlace químico, y comencé a evidenciar que muchas de estas restricciones que son presentadas por los artículos las presentamos nosotros los estudiantes de magisterio. Considero que esta especie de conocimiento es un elemento clave en el diseño de la ReCo y, más que en el diseño de la ReCo es un elemento clave al momento de ir a ejercer mi profesión. (Entrevista, Yuly)

Las anteriores actividades de formación les brindaron la oportunidad a los profesores en formación de comenzar a identificar y desarrollar este componente del CPC de la química. En consecuencia lograron un conocimiento más profundo de los temas alrededor de la comprensión de los estudiantes, el cual se puso de manifiesto en las celdas del ítem en cuestión. Así, en las últimas ReCo se puede ver que los futuros profesores comenzaron a considerar la diferenciación e integración de los tres niveles de representación como un elemento que desempeña un papel clave en la comprensión conceptual e integrada del currículo de la química, dado que evita que la memoria de trabajo de los estudiantes se sobrecargue y se genere un rompimiento entre la información nueva y la que ya reside en la memoria permanente del sujeto.

Otra restricción que los profesores en formación comenzaron a identificar hace referencia a la naturaleza abstracta de los diferentes tópicos del currículo de la química. Desde luego, estos conceptos no pertenecen al mundo concreto, sino que son una construcción del intelecto humano a partir de la interpretación de los datos generados desde los diseños experimentales. Es decir, la química está construida a partir de una serie de modelos teóricos que no pertenecen a las coordenadas del aquí y el ahora. Este aspecto hace que el aprendizaje de estos modelos sea de una gran dificultad, razón por la cual se deben representar a través de analogías y modelos que tienen como fin reducir esa naturaleza abstracta. 
Este presupuesto se puede evidenciar en el ítem 4 de la ReCo sobre la gran idea "investiga sobre las aplicaciones de los fluidos supercríticos y sus ventajas desde el punto de vista ambiental":

Dado que el fenómeno de los fluidos supercríticos no se puede visualizar de forma fácil y natural, entonces se trabajar con los conceptos, a partir de gráficas, imágenes y videos. Si bien, se modelizan y representa de una manera apropiada dicho fenómeno, aún sigue siendo una representación, por lo que los estudiantes difícilmente tienen un contacto concreto con dicho fenómeno. Lo anterior, de la mano con lo complejo del concepto, le otorga alto grado de restricción al mismo, por lo que es muy difícil para el estudiante poder imaginar y representar dicho fenómeno. (ReCo, Vanessa).

\section{Conocimiento y creencias acerca de la evaluación en la alfabetización científica}

Los profesores en formación representan las estrategias de evaluación de las grandes ideas que configuran el tópico en consideración en términos generales. Es decir, las formularon haciendo referencia a aspectos conceptuales, procedimentales y actitudinales en un nivel de orden genérico; sin embargo, no enunciaron cuáles serían los conceptos, las habilidades y las actitudes a monitorear en un nivel de detalle más fino a lo largo del proceso de enseñanza y aprendizaje del tópico específico. Dicha situación se observa en un fragmento de la ReCo de Vanessa que hace referencia a la evaluación:

Durante todo el desarrollo de las actividades de aprendizaje los estudiantes constantemente socializan en grupos de discusión, lo cual posibilita no solo el aprendizaje cooperativo, sino que además fortalece los argumentos que se exponen y ayuda a generar interpretaciones más generales y en concordancia a [sic] los argumentos científicos. (ReCo, Vanessa)

Se destaca que la estrategia de socializar el desarrollo de las ReCo diseñadas por los respectivos pares de profesores en formación les brinda la oportunidad de comenzar a mover su pensamiento y acciones sobre las estrategias de evaluación desde una perspectiva general de la química hacia una articulada con el tópico específico, en la cual el diseñador de la ReCo formula las diferentes actividades de evaluación alineadas con cada una de las ideas que constituyen el concepto en cuestión. Esta situación le permite al profesor monitorear el nivel de comprensión y confusión sobre la gran idea que tienen los estudiantes durante la transacción instruccional, con el propósito de brindar retroalimentación desde una perspectiva socrática en el momento en que detecte los eventos críticos. Esta afirmación se puede observar en una sección de la ReCo final propuesta por Yuly.

Un factor crítico en la nueva concepción de evaluación que comenzaron a construir los profesores en formación es la que hace referencia a esta como un proceso transversal y dinámico en la enseñanza y aprendizaje de un tópico específico. De ahí que los profesores en formación en su ReCo final dejan ver que la enseñanza de los contenidos la estructuran en tres fases: exploración, introducción y aplicación. La primera permite determinar las concepciones alternativas con las que llegan los estudiantes al aula de química; la segunda, posibilita que evolucionen estas concepciones hacia los modelos teóricos de la química; y por último, estos nuevos conocimientos se implementan en otras situaciones. 
En este sentido, el monitoreo del nivel de comprensión y confusión se da a lo largo de todo el proceso con el fin de llevar a cabo una evaluación formativa. De hecho, cuando el profesor detecta un incidente crítico les formula a los estudiantes unos interrogantes que están dentro de la zona de desarrollo proximal, esto permite la transacción de significado con el propósito de superar las dificultades. Es decir, no les soluciona el inconveniente a los estudiantes sino que les genera la necesidad de aprender. Sin embargo, este tipo de evaluación no excluye a la acreditativa que se da al final de cada lección, lo importante es que esta no se convierte en el foco de valoración. Estos presupuestos se pueden evidenciar en el diario reflexivo de una de las profesoras en formación:

... El docente primero debe dar todas las instrucciones para luego pasar a la estructura de clase de pequeños grupos de discusión. A partir de las siguientes preguntas se busca que los estudiantes expliciten sus ideas sobre esta temática, para que el docente guíe adecuadamente las actividades con el fin de que sus concepciones se ajusten a las ideas de la ciencia... (Diario reflexivo, Vanessa).

\section{Conclusiones}

Los resultados de esta investigación han permitido ver que el curso Problemas de la Enseñanza y el Aprendizaje de la Química por Orientación Reflexiva, que se centra en el diseño autónomo de una ReCo de un tópico específico, es una estrategia óptima para ayudar a los profesores en formación para empezar a identificar, explicitar y desarrollar el conocimiento pedagógico del contenido hipotético. Así pues, este les dio unas bases de conocimiento que les ayudaron a mejorar la confianza y capacidad para ubicar, seleccionar y determinar información relevante con el fin de diseñar una ReCo a pesar de su falta de experiencia de enseñanza.

Por supuesto, dicha estrategia de desarrollo profesional cumplió el papel de catalizador de los procesos de razonamientos y acciones de los profesores en formación, que recogen y amalgaman los elementos del contenido, el estudiante y la enseñanza con el fin de diseñar ambientes de aprendizaje de un tópico específico. Esta situación les permitió moverse de manera progresiva desde un punto donde solo se limitaron a enumerar de manera desarticulada una serie ideas que configuran el contenido a enseñar, sin vincularlas con unas metas de aprendizaje claras, a un estado donde seleccionaron, secuenciaron y formularon las ideas claves que configuran el contenido en cuestión.

Conviene subrayar que el desarrollo de esta herramienta metodológica ( $\mathrm{ReCo}$ ) les posibilitó a los profesores en formación comenzar a visualizar el currículo horizontal y vertical de la química. Así, esta meta de desarrollo profesional fue catalizada por la solución que le dieron al ítem n. ${ }^{\circ} 3$ de la ReCo (¿̇Qué más sabe respecto a esta idea (y qué no incluye en sus explicaciones a sus alumnos)?), la cual fue discutida de forma colegiada. De hecho, por medio de dicha tarea los futuros profesores comenzaron a establecer relaciones estrechas entre las 
ideas que constituyen el contenido en cuestión y la serie de tópicos de otras lecciones que se abordan dentro y a lo largo de los grados de la escuela secundaria. Es decir, empezaron a configurar el diseño y la enseñanza del currículo de la química como un sistema complejo, y no como un conjunto de ideas y propósitos desarticulados.

Otro aspecto relevante de la identificación, explicitación y desarrollo del CPC de la química de los profesores en formación hace referencia a la toma de decisiones instruccionales. Desde luego, estas interaccionan de forma bidireccional con las decisiones curriculares buscando iluminar las intenciones de diseño que se traducen en una secuencia de actividades de aprendizaje, cuyo fin central es el de generar el escenario que medie la comprensión conceptual e integrada de un tópico del currículo de la química en unos estudiantes singulares.

Se destaca que el diseño de la ReCo brinda la posibilidad para que los profesores en formación desarrollen una articulación estrecha y consciente entre las teorías del aprendizaje, la naturaleza del tópico específico y el problema de enseñanza. Es decir, el desarrollo progresivo de las ReCo les permitió comenzar a ver que aquellos conceptos del currículo de la química cuyo nivel de abstracción es alto y para los cuales no se encuentran situaciones del mundo de la vida que los represente, deben de ser formulados para la enseñanza a través de representaciones (animaciones, simulaciones, video, entre otros) que articulen la apariencia, la representación y el fenómeno natural. En efecto, dicha articulación les ayudó a los profesores en formación a iluminar la toma de decisiones en cuanto a las técnicas, estrategias y modelos de enseñanza de las ciencias que estuvieran más alineadas con la naturaleza del contenido y el problema de su enseñanza.

Por todo lo anterior, los profesores en formación empezaron a considerar que para poder alcanzar las metas del diseño de la enseñanza de un contenido de la química, se debe vincular la identificación y comprensión profunda de las ideas claves que configuran el tópico en cuestión con aspectos tales como: justificación de las ideas seleccionadas; dificultades que los estudiantes podrían encontrar en el aprendizaje de estas ideas; concepciones alternativas; formas de representar y formular cada una de las ideas seleccionadas; técnica, estrategias y modelos de enseñanza de la química; secuencias de actividades de aprendizaje; y estrategias instruccionales alineadas con las metas de aprendizaje. Desde luego, para completar esta tarea de diseño se requirió que dichos profesores estuvieran familiarizados con el contenido, las fuentes y la justificación del porqué de dicha selección.

Finalmente, los investigadores consideran que el diseño de la ReCo como una herramienta para identificar, explicitar y desarrollar los componentes del CPC es por una tarea que implica un alto nivel de cognición y trabajo. No obstante, en este estudio se evidenció que un andamiaje cuidadoso de las experiencias de aprendizaje como las ilustradas desde los cuatro "ámbitos de reflexión" arriba mencionados, brinda a los profesores en formación una valiosa oportunidad para comenzar acceder a la especie de conocimiento y pensamiento que posee un profesor experto y ejemplar, sin llegar a sentirse abrumados por los requerimientos del diseño y la enseñanza de los tópicos del currículo de la química. 


\section{Referencias bibliográficas}

Abell, K. y Bryan, L. A. (1997). Reconceptualizing the elementary science methods course using a reflection orientation. Journal of Science Teacher Education, 8 (3), 153-166.

Bertram A. y Loughran, J. (2012). Science teachers' views on CoRes and PaP-eRs as a framework for articulating and developing pedagogical content knowledge. Research in Science Education, 42, 1027-1047.

Candela, B. F. (2012). La captura, la documentación y la representación del CPC de un profesor experimentado y "ejemplar" acerca del núcleo conceptual de la discontinuidad de la materia (tesis de maestría). Universidad del Valle, Cali.

Candela, B. F. y Viafara, R. (2014b). Aprendiendo a enseñar química: la CoRe y los PaP-eR como instrumento para identificar y desarrollar el CPC. Programa editorial de la Universidad del Valle. Cali.

Candela, B. F. y Viafara, R. (2014a). Articulando la CoRe y los PaP-eR al programa educativo por orientación reflexiva: una propuesta de formación para el profesorado de química. Tecné, Episteme y Didaxis: TED, 35, 89-111.

Christensen, T. K. (2008). The role of theory in instructional design: Some views of an ID practitioner. Performance Improvement, 47(4), 25-32.

Cess-Newsome, J. (1999). Secondary teachers' knowledge and beliefs about subject matter and their impact on instruction. En Cess-Newsome J. y N. G. Lederman (eds.). PCK and Science Education. Netherlands: Dordrecht: Kluwer Academic Publishers. Recuperado de http://link.springer.com/chapter/10.1007\%2F0-30647217-1_3\#page-2

Hume, A. (2010). CoRes as Tools for promoting Pedagogical Content Knowledge of novice science teachers. Chemistry Education in New Zealand, 119, 13-19.

Hume, A. (2011). Using collaborative CoRe design in chemistry education to promote effective partnerships between associate and student teachers. Chemistry Education in New Zealand, 13-20.

Hume, A. y Berry, A. (2013). Enhancing the practicum experience for pre-service chemistry teachers through collaborative CoRe design with mentor teachers. Research in Science Education, 43, 2107-2136

Loughran, J.; Berry, A. y Mulhall, P. (2006). Understanding and developing science teachers' pedagogical content knowledge. Rotterdam: Sense.

Loughran, J.; Mulhall, P. y Berry, A. (2004). In search of pedagogical content knowledge in science: Developing ways of articulating and documenting professional practice. Journal of Research in Science Teaching, 41 (4), 370-391. 
Loughran, J.; Mulhall, P. y Berry, A. (2008). Exploring pedagogical content knowledge in science teacher education. International Journal of Science Education, 30(10), 1301-1320.

Magnusson, S.; Krajcik, J. y Borko, H. (1999). Nature, sources and development of pedagogical content knowledge. En J. Cess-Newsome \& N. G. Lederman (eds.). Examining pedagogical content knowledge (pp. 95-132). Dordrecht: Kluwer Academic Publishers.

Mora, W. y Parga, D. (2008). El conocimiento didáctico del contenido en química: integración de las tramas de contenido/histórico-epistemológicas con las tramas de contexto. Tecné, Episteme y Didaxis, TED, 24, 56-81.

Mulhall, P.; Berry, A. y Loughran, J. (2003). Frameworks for representing science teachers' pedagogical content knowledge. Asia-Pacific Forum on Science Learning and Teaching, 4(2), 1-25.

Shulman, L. (1986). Those who understand: Knowledge growth in teaching. Educational Researcher, 15(2), 4-14.

Shulman, L. (1987). Knowledge and teaching: Foundations of the new reform. Harvard Educational Review, 57(1), 1 - 22.

Stake, R. (1999). Investigación con estudio de casos. España: Morata.

Strauss, A. y Corbin, J. (2002). Bases de la Investigación cualitativa: técnicas y procedimientos para desarrollar la teoría fundamentada. (E. Zimmerman, trad.). Medellín: Facultad de Enfermería de la Universidad de Antioquia. (Trabajo original publicado en 1990). 


\section{Anexo 1. Tabla de la herramienta metodológica de la ReCo}

UNIVERSIDAD DEL VALLE

INSTITUTO DE EDUCACIÓN Y PEDAGOGÍA

MAESTRIA EN EDUCACIÓN (ÉNFASIS EN ENSEÑANZA DE LA CIENCIA)

BASE PARA LA ENTREVISTA

¿Cuáles son las ideas científicas que se encuentran en el centro del tópico "Enlaces químicos" o "Propiedades de sólidos, líquidos y gases? Es decir, seleccione entre tres a cinco ideas en las que acostumbre a dividir la enseñanza del concepto de la discontinuidad de la materia. Se trata de que en ese conjunto de ideas estén reflejadas las más importantes del tema a impartir, o de sus precedentes.

Para cada una de estas ideas responda las siguientes preguntas:

\begin{tabular}{|c|c|c|c|}
\hline \multicolumn{4}{|l|}{ IDEAS/CONCEPTOS IMPORTANTES EN CIENCIAS } \\
\hline & Idea n. ${ }^{\circ} 1$ & Idea n. ${ }^{\circ}$ & Idea n. ${ }^{\circ} 3$ \\
\hline \multicolumn{4}{|l|}{ 1. ¿Qué intenta que aprendan los alumnos alrededor de esta idea? } \\
\hline \multicolumn{4}{|l|}{ 2. ¿̇Por qué es importante que los alumnos sepan esta idea? } \\
\hline \multicolumn{4}{|l|}{$\begin{array}{l}\text { 3. ¿̇ué más sabe respecto a esta idea (y qué no incluye en sus explicaciones a } \\
\text { sus alumnos)? }\end{array}$} \\
\hline \multicolumn{4}{|l|}{$\begin{array}{l}\text { 4. ¿̇Cuáles son las dificultades/limitaciones relacionadas con la enseñanza de } \\
\text { esta idea? }\end{array}$} \\
\hline \multicolumn{4}{|l|}{$\begin{array}{l}\text { 5. ¿̇Qué conocimientos acerca del pensamiento de los alumnos influyen en su } \\
\text { enseñanza de esta idea? }\end{array}$} \\
\hline \multicolumn{4}{|l|}{ 6. ¿Qué otros factores influyen en su enseñanza de esta idea? } \\
\hline \multicolumn{4}{|l|}{$\begin{array}{l}\text { 7. ¿Cuáles procedimientos de enseñanza emplea? (y las razones particulares de } \\
\text { su uso con esta idea). }\end{array}$} \\
\hline $\begin{array}{l}\text { 8. ¿̇Qué formas específicas de evaluación del entendimiento o de la confusión de } \\
\text { los alumnos emplea alrededor de esta idea? }\end{array}$ & & & \\
\hline
\end{tabular}

Anexo 2. Encuesta sobre el diseño de la ReCo

Universidad del Valle

Instituto de Educación y Pedagogía

Maestría en Educación (Énfasis en Ciencias Naturales)

Cuestionario

1. ¿Qué implicó la tarea de la ReCo para usted?

2. ¿Qué desearía hacer con esta ReCo y cuándo?

3. ¿Cuál fue el propósito del diseño de la ReCo para el proyecto?

4. ¿Cómo llevó a cabo el diseño de su ReCo?

5. ¿Conocía el tema a diseñar a través de la ReCo y en qué profundidad? 
6. ¿Cuál fue el elemento más problemático dentro del diseño de la ReCo y el más sencillo y por qué?

7. Si usted no estuviera usando el enfoque de la ReCo, żcómo podría preparar la enseñanza de un tópico de la química?

8. ¿Cómo el diseño de la ReCo informa su pensamiento acerca de la práctica de enseñanza?

9. ¿̇u pensamiento acerca de los elementos del CPC ha cambiado como resultado del diseño de la $\mathrm{ReCo}$ y por qué?

10. ¿Qué beneficios, ideas y ventajas trajo el proceso de diseñar la ReCo?
1 1. ¿Volvería a usar la ReCo para diseñar la enseñanza de un tema? ¿̇Por qué?

12. ¿̇Cuáles aspectos de su conocimiento profesional se generaron a través del diseño de la ReCo?

13. ¿Cuál ha sido el desarrollo de su CPC durante esta investigación?

14. ¿̇Puede usted ver alguna articulación entre el CPC y su ReCo?

15. ¿̇Qué significa para usted la ReCo?

16. ¿̇Puede ver usted alguna articulación entre el CPC, la ReCo y el desarrollo de su conocimiento profesional acerca de la enseñanza. 